\title{
Drug Delivery Tailored for the Need-Case Series of Oral Lichen Planus
}

\author{
Archana Venugopal, T.N. Uma Maheswari* \\ Department of Oral Medicine and Radilogy, Saveetha Dental College, Chennai, Tamil Nadu, INDIA.
}

\begin{abstract}
Lichen planus is an inflammatory mucocutaneous disorder. The pathogenesis of lichen planus is predominantly $T$ cell mediated. The condition is associated with it are hepatitis $\mathrm{C}$, graft versus host disease, medications [antiarrythmic drugs, anti-hypertensive], dental materials [amalgam restoration] etc. Immune modulators have been widely used in the management of lichen planus. Corticosteroids are the most widely used among them. There is various mode of delivering corticosteroids in oral lichen planus. The systemic administration is reported to cause more adverse drug reaction when compared to local drug delivery systems. The case series describes three cases of lichen planus at different sites. It also high lights the site-specific treatment applied in them.
\end{abstract}

Key words- Lichen planus, Local drug delivery, Corticosteroids, Systemic steroids.

Key message: Primary care dentist should be aware of site specific drug delivery systems available for the treatment of Oral Lichen Planus.

Correspondence

Prof. T.N. Uma Maheshwari, Department of Oral Medicine and Radiology, Saveetha Dental College, Chennai, Tamil Nadu, INDIA.

Phone: 9840958339

Email: umasamsi@gmail.com

DOI: 10.5530/jyp.2018.10.55

\section{INTRODUCTION}

Lichen Planus is defined as the chronic inflammatory disorder affecting the skin, mucus membrane, nail and scalp. It can be clinically classified as Reticular, atropic, plaque like, bullous and erosive type. Erosive lichen planus has increased chance of malignant transformation. The range of its malignant transformation is between $0.04 \%-1.74 \% .{ }^{1}$ It frequently affects women more than men in the ration 1.4:1. ${ }^{2}$

The pathogenesis of Oral lichen planus antigen expression by the keratinocytes or unmasking the antigen of heat shock protein or self-antigen. These antigens presented to the T cells causes apoptosis of the basal cells of the epithelium. More CD4 and CD8 T cells migrate to the site. This migration is caused by the antigen binding to the major histocompatibility complex [MCH -I]. MCH- II expression increases the langrahan cells in the lesion. Increased antigen presentation activates CD4 and CD8 cells and these cells in turn causes killing of basal keratinocytes and apoptosis. ${ }^{3}$ This causes degeneration of the basement membrane and lymphocytic infiltration of the connective tissue adjacent to the basement membrane.

The management of oral lichen planus include corticosteroids [triamcinolone, prednisone, clobetasol etc], calciurin inhibitors (tacrolimus, cyclosporine), mycophenolate mofetil, azathioprine and methotraxate. ${ }^{1}$

The case series describes the 3 different cases requiring different means of drug delivery in oral lichen planus. There by describing the effectiveness of using corticosteroid by overcoming its adverse effect.

\section{CASE 1}

A 57 years old female patient reported to the OPD with the chief complaint of burning sensation of the oral mucosa for past 1 month. The burning sensation was generalized, it aggravated on taking spicy food. Patients past medical history and past dental history did not have significant history. On clinical examination diffused regions of erythema was seen in the right alveolar mucosa [Figure 1] and left buccal mucosa [Figure 2]. These lesions also showed interlacing white striae, surrounding them. The patient was provisionally diagnosed with Reticular lichen planus. The patient was advised $1 \%$ Triamcinolone acetonide for topical application thrice daily for 3 weeks. Patient was reviewed every week. As the lesion slowly healed the frequency of topical application was reduced and tapered.

\section{CASE 2}

A 52 years old male patient reported with severe burning sensation of the buccal mucosa, gingival for past 3 months. This was associated with bleeding on brushing the teeth. History revealed patient to be diabetic and hypertensive for past 6 years and 8 years respectively. The patient was on Atenolol $50 \mathrm{mg}$, once a day and Metformin 500mg twice a day.

On clinical examination erythematous soft friable gingival along the marginal gingival was seen along the teeth of the upper arch. [Figure 3] The buccal mucosa in relation to 36,37 and 38 also showed irregular shaped ulceration surrounded by erythema and white interlacing lines or wickam striae. From the clinical examination and history, the patient was provisionally diagnosed with Grinspan syndrome.

An occlusal soft split covering the gingival was fabricated. Patient was advised to apply $1 \%$ triamcinolone acetonide on the occlusal split and wear the split for 10 to 15 min three times a day. topical application of triamcinolone was with cotton on the buccal mucosa and tongue was advised. Patient was asked to rinse 10 to $15 \mathrm{~min}$ and advised not to drink or eat for next $30 \mathrm{~min}$. Patient continued the medication for 2 weeks and was reviewed. The lesion had healed significantly. Later the frequency of 

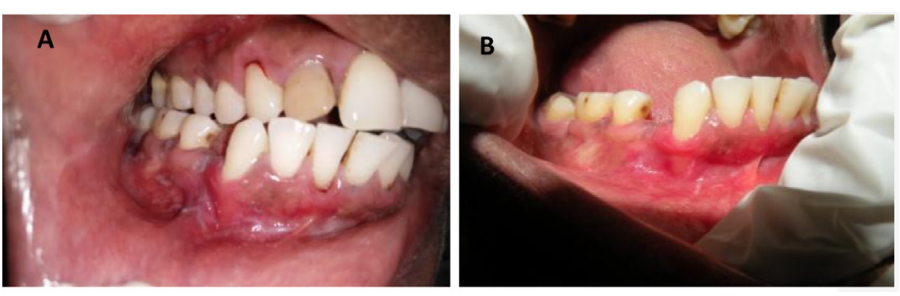

Figure 1: A. Alveolar mucosa showing reticular lichen planus, B. Completely healed lesion in the region of alveolar mucosa.
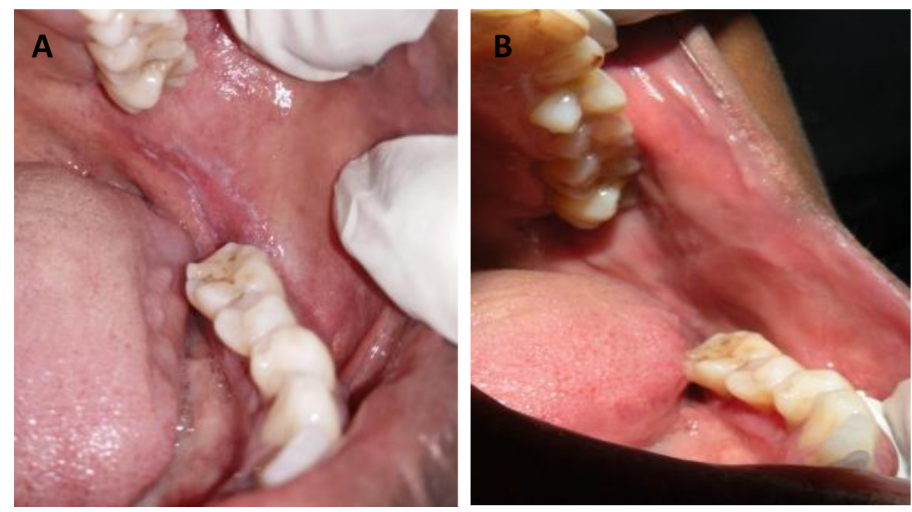

Figure 2: A. Left buccal mucosa showing reticular lichen palnus. B. Completely healed lesion in the region of left buccal mucosa.

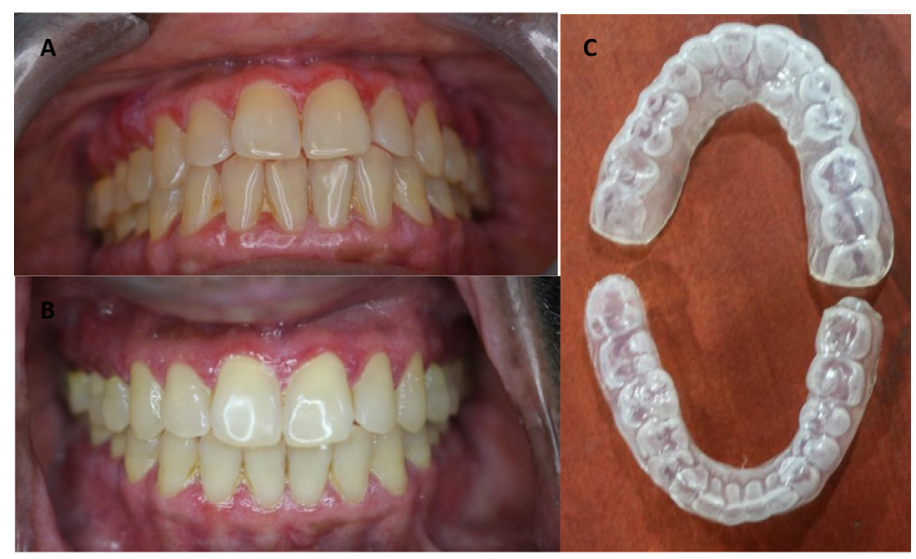

Figure 3: A- Erythematous, soft marginal gingiva showing desquamation. B- Shows healed lesions in the gingival. C- Occlusal splint used for drug delivery system.

the medication was reduced to taper the dosage for the consecutive week and then stopped slowly.

\section{CASE 3}

A 54 years old female patient reported with the chief complaint of burning sensation and pain in the buccal mucosa, labial mucosa and tongue for past 3 months. The burning sensation is associated with severe ulceration. History of present illness revealed a similar pruritic, purple plaque on the right inner thigh region, which occasionally appears and disappears. History revealed she was neither diabetic, hypertensive or under any systemic illness. On clinical examination diffused regions of ulceration surrounded by regions erythema and whitish striae was seen in the right and left buccal mucosa [Figure 4], lower labial mucosa and palate. These regions were interspersed with hyper pigmented patches around the lesions. The tongue also showed hyper pigmented patch with whitish plaque like keratotic regions on the dorsal surface of the tongue. The lesion in the right inner thigh was an irregular flattened purplish plaque of size $3.4 \times 2.3 \mathrm{~cm}$.

From the history and clinical examination, the patient was provisionally diagnosed with erosive lichen planus. An incisional biopsy of was taken from the right buccal mucosa. The histopathology [Figure 5] revealed hyper parakeratinized stratified squamous epithelium of variable thickness with few areas showing saw tooth shaped rete ridges and several areas of basal cell degeneration. Underlying connective tissue showed moderate vascularity. The histopathology was suggestive of lichen planus.

The weight of the patient was measured, the blood sugar level and the blood pressure value where noted before initiation of the treatment. $0.05 \%$ clobetasol propionate ointment for topical application twice daily for and single morning dose of $5 \mathrm{mg}$ prednisolone tablet, was advised for one week followed by $10 \mathrm{mg}$ single dose of Prednisolone tablet was advised. Patient continued the same medication for a period of 2 weeks and then the $10 \mathrm{mg}$ prednisolone was tapered to $5 \mathrm{mg}$ for next 1 week. The following week patient was advised to taper the dose to $5 \mathrm{mg}$ prednisolone tablet once in 2 days and then stopped, patient however continued the topical steroid for 5-week period. Patient was reviewed every week,

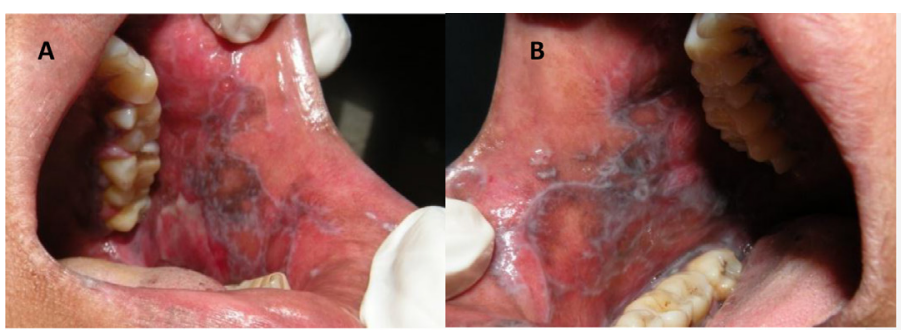

Figure 4: Right and Left buccal mucosa showing severe ulcerations with interlacing white striae.

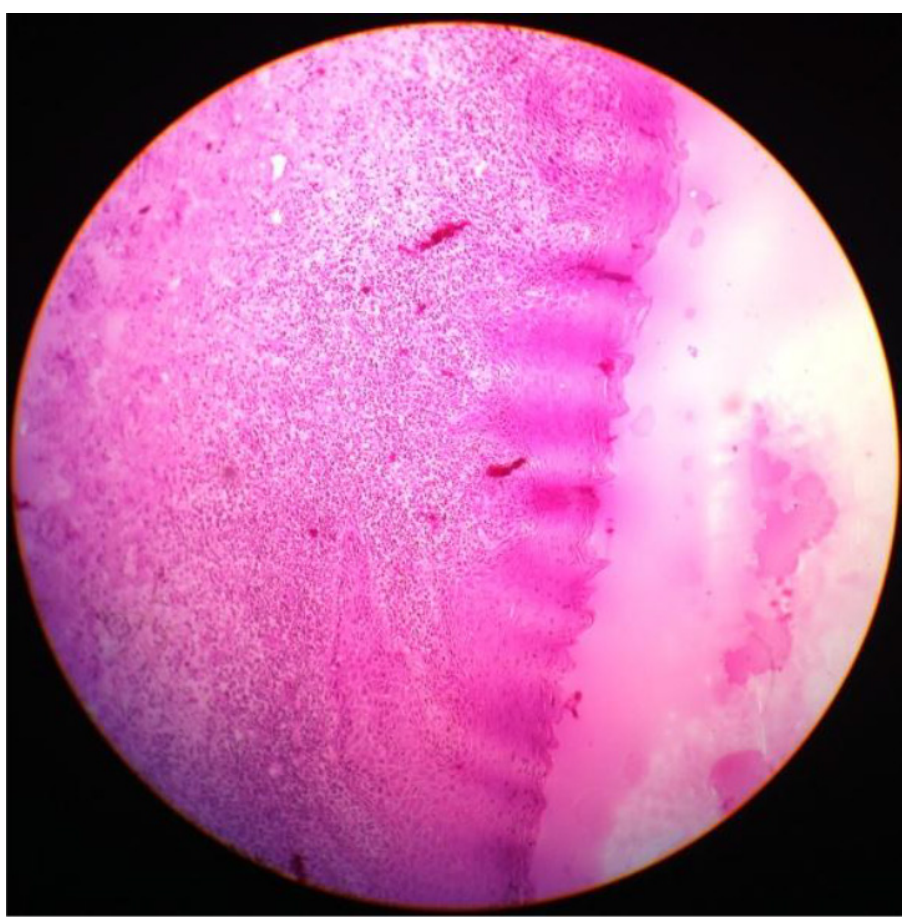

Figure 5: Photomicrograph showing hyperparakeratinized stratified squamous epithelium with saw tooth shaped rete ridges. 


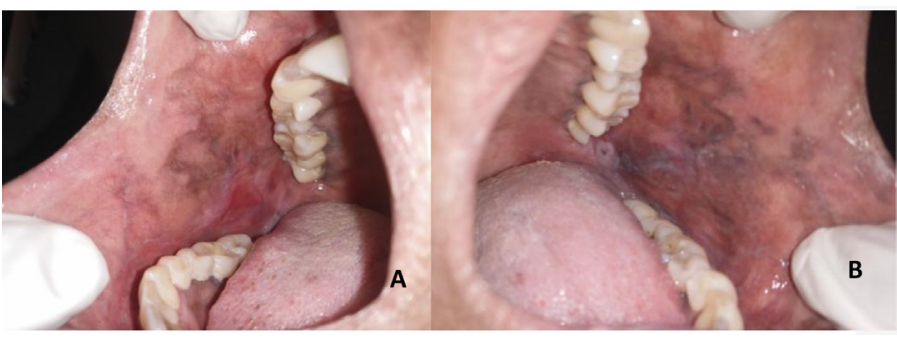

Figure 6: Healed lesion on the right and left buccal mucosa after 5 weeks of treatment.

by end of $5^{\text {th }}$ week the lesion and the burning sensation was reduced considerably. [Figure 6] The patient was reviewed every month for 6 months.

\section{DISCUSSION}

As lichen planus a chronic inflammatory mucocutateous disorder, it affects the basal cells and the adjacent connective tissue, the immune activity occurs principally in the upper part of the connective tissue. From the above nature of the disease it is made evident that the drug used for its treatment should not only cross the permeability barrier but also be retained in the basal layer and the connective tissue. ${ }^{4}$ This proves the fact that local drug delivery is more effective in treatment of Oral lichen planus.

The drug delivered should not be lost in the systemic circulation unless the disease is of extensive nature. The major disadvantage of systemic delivery is side effects of the drugs and systemic loss of drug in the body. Corticosteroid with its predominant anti-inflammatory action is first line drug in treating oral lichen planus. ${ }^{5}$ The main anti-inflammatory effect of steroids include, decreased phagocytosis, antigen presentation, impaired migration of phagocytes and polymorphonuclear leukocytes, suppressed histamine and kinin release and inhibition of A2 thus reducing inflammatory mediator production. ${ }^{6}$

The adverse effects of corticosteroid include peptic ulceration, cushing's disease diabetes, cardiovascular effects like sodium retention and increased intra vascular volume thereby causing hypertension. The other adverse effects are cataracts, growth retardation in children, osteoporosis, infections and malignancies like lymphomas. All these adverse events are more in systemic administration when compared to local drug delivery.

Literature states topical drug penetrates, ulcerated or eroded mucosa more readily then intact healthy mucosa. This adds to the advantage of using local drug delivery as a mean of treating lichen planus. There are three methods of diffusion of the drugs in local drug delivery system, which are 1. Passive diffusion, 2. Carrier mediated transport and 3. Endocytosis and exocytosis by the cell. ${ }^{4}$ Corticosteroid formulations primarily follows passive diffusion, where the lipophilic molecules diffuses through trans cellular and para cellular means. ${ }^{6}$

Systemic steroids are second line of treatment when the topical steroids are not effective. ${ }^{7}$ these are employed in lowest possible dose. They are termed double "edged sword", as they have both therapeutic benefits and adverse effects. ${ }^{8}$ To attenuate the adverse effects it is advised to go for early morning dosing scheme as they replicate the normal rhythm of the body. Tapering of the steroids should be started as soon as possible. If treatment lasts for more than 1 to 3 weeks a gradual tapering depending on patient's general health is advised. If treatment lasts for 1-week steroid can be stopped without tapering. In lesions requiring high doses of steroids for its control, other immune modulators are preferred. ${ }^{6}$

In the Case 1 the lesion is a milder form of reticular lichen planus seen in the alveolar mucosa and the buccal mucosa so a medium potent steroid, triamcinolone acetonide $1 \%$ oral gel was preferred for topical use. In the Case 2 the lesion was more extensive on the gingiva than in the buccal mucosa, so it was essential for the medication to be retained longer in the gingival lesion, so a local drug delivery system [Occlusal Split with the gingival extension] was preferred for the topical application of the medium potent steroid triamcinolone acetonite $1 \%$ Oral gel. This system prevented the steroid from getting washed away by the saliva and retained it longer in the site. In the Case 3 the lesion was extensive and severe in the buccal mucosa, labial mucosa and palatal mucosa. It also had cutaneous lesion so along with very high potent steroid, Clobetasol propionate $0.05 \%$ cream for topical application, low dose of Prednisolone was preferred treatment. Another commonly used mode of delivering corticosteroids in lichen planus is intra lesionally. The commonly used agents are Prednisolone and Triamcinolone. ${ }^{9}$

\section{CONCLUSION}

Each patient is unique so are their illness. It is the responsibility of the dentist to decide on the method of delivery of the prescribed drug. While planning the treatment for patient's oral condition, his general health should also be taken into consideration. Dentist's extensive knowledge in tailoring the treatment will prove to be effective in successful outcome.

\section{CONFLICT OF INTEREST}

The authors declare no conflict of interest.

\section{REFERENCES}

1. De Rossi SS, Ciarrocca K. Oral lichen planus and lichenoid mucositis. Dent Clin North Am. 2014;58(2):299-313.

2. Lavanya N, Jayanthi P, Rao UK, Ranganathan K. Oral lichen planus: An update on pathogenesis and treatment. J Oral Maxillofac Pathol JOMFP. 2011;15(2):127-32.

3. Alrashdan MS, Cirillo N, McCullough M. Oral lichen planus: A literature review and update. Arch Dermatol Res. 2016;308(8):539-51.

4. Sankar V, Hearnden V, Hull K, Juras DV, Greenberg MS, Kerr AR, et al. Local drug delivery for oral mucosal diseases: Challenges and opportunities. Oral Dis. 2011;17(s1):73-84.

5. Cheng S, Kirtschig G, Cooper S, Thornhill M, Leonardi-Bee J, Murphy R. Interventions for erosive lichen planus affecting mucosal sites. Cochrane Database Syst Rev. 2012;15(2):CD008092.

6. Georgakopoulou EA, Scully C. Systemic use of non-biologic corticosteroids in orofacial diseases - Georgakopoulou - 2013 - Oral Diseases. 2014;20(2):127-35. Wiley Online Library Internet]. Cited 2017 Nov 12]. Available from: http://onlinelibrary.wiley.com/doi/10.1111/odi.12132/abstract

7. Thongprasom K, Dhanuthai K. Steriods in the treatment of lichen planus: A review. J Oral Sci. 2008;50(4):377-85.

8. Sanghavi J, Aditya A. Applications of Corticosteroids in Dentistry. J Dent Allied Sci. 2015;4(1):19-24.

9. Scully C. Oral and Maxillofacial Medicine-The basis of diagnosis and treatment. Edition 3. Edinburg, London: Elsevier Health Sciences. 2008.

Article History: Submission Date : 19-11-2017 ; Revised Date : 09-12-2017; Acceptance Date : 27-01-2018.

Cite this article: Venugopal A, Maheswari TNU. Drug Delivery Tailored for the Need- Case Series of Oral Lichen Planus. J Young Pharm. 2018;10(2):246-8. 